\title{
Expressive Models for Synaptic Plasticity
}

\author{
Andrea Bracciali ${ }^{1}$, Marcello Brunelli ${ }^{2}$, Enrico Cataldo ${ }^{2}$, and Pierpaolo Degano ${ }^{1}$ \\ 1 Dipartimento di Informatica, Università di Pisa. \\ 2 Dipartimento di Biologia, Università di Pisa. \\ braccia@di.unipi.it, mbrunelli@biologia.unipi.it, ecataldo@biologia.it, \\ degano@di.unipi.it
}

\begin{abstract}
We explore some presynaptic mechanisms of the calyx of Held synapse through a stochastic model. The model, drawn from a kinetic approach developed in literature, exploits process calculi as formal grounds, enjoys nice compositional properties, has a direct computational implementation that supports simulation trials, and, to our knowledge, represents the first process calculi based model of a presynaptic terminal. Simulation results have shown coherence with experimental data and robustness against sensitivity analysis. The core model has been extended in order to address some issues related to open problems: we discuss hypotheses on short-term synaptic enhancement (facilitation) and depression, i.e. plasticity mechanism that are related to memory and learning. The two aims of our work, i.e. addressing neural mechanisms and validating and possibly improving, process calculi based modeling techniques are discussed throughout the paper, together with the results of experiments.
\end{abstract}

\section{Motivations}

Research in life sciences is benefiting from a large availability of formal description techniques and analysis methodologies. These allow both the phenomena investigated to be precisely modeled and virtual experiments to be performed in silico. Such experiments may result in easier, faster, and satisfying approximations of their in vitro/vivo counterparts. A promising approach is represented by the study of biological phenomena as a collection of interactive entities through process calculi equipped with stochastic semantics. These exploit formal grounds developed in the theory of concurrency in computer science, account for the not continuous, nor discrete, nature of many phenomena, enjoy nice compositional properties and allow for simulations that have been demonstrated to be coherent with data in literature. The huge amount of information produced in the field of neurobiology and the complex dynamics of the biological processes require the utilization of mathematical and computational modeling methods [KS98,FMWT02]. Neurons represent the elementary components of the nervous systems, able to communicate with each other at highly specialized contact sites called synapses. In general, each neuron consists of a somatic cellular body, on which a variable number of thin elongated structures called dendrites 
converge and from which a long single structure called axon emerges, branching in several synaptic terminals. The synaptic terminals of the transmitting neuron (the presynaptic element) send signals by releasing chemical molecules (neurotransmitters) to the dendritic part of the receiving neuron (postsynaptic term) $[\mathrm{RBM}]$.

The synapses are the places of functional contacts between neurons, where the information is stored and transmitted from one to another neuron. Synaptic transmission is a complex process and current knowledge on synapses is based on the analysis of a limited number of experimental synaptic models $\left[\mathrm{FGJ}^{+} 06\right]$. Synaptic transmission involves the presence of calcium ions in the presynaptic terminal, which control the transmitter release process [ZKS04], consisting in the exocitosis of synaptic vescicles (small elements containing the neurotransmitters) located at the presynaptic so-called active zone [Sud04].

The electrical signals (action potentials) arriving at the synaptic terminal induce the opening of the $\mathrm{Ca}^{2+}$ channels. The transient elevation of the internal $\mathrm{Ca}^{2+}$ concentration in the presynaptic terminal triggers synaptic vescicle exocitosis, and hence the neurotransmitter release (calcium-triggered-release hypothesis). Interestingly, chemical messengers (intracellular) and modulators (extracellular) regulate the relationship between action potential and release in a synaptic terminal, which is also altered by the repeated activity. All these things make the presynaptic terminal a kind of computational unit, which changes its output based on its previous activity and ongoing modulation.

Theoretical and functional studies have suggested that $\mathrm{Ca}^{2+}$ acts on presynaptic vescicles by a local huge and short-lived elevation of its concentration. The locality and rapidity of the concentration variation render the study of this phenomenon not approachable with the conventional microscopic imaging techniques. Among the methods envisaged to overcome this limitations, one very fruitful is the so-called reverse approach, in which $\mathrm{Ca}^{2+}$ uncaging is induced in the presynaptic element. The uncaging method induces spatially homogeneous $\mathrm{Ca}^{2+}$ elevation, implying that measuring the $\mathrm{Ca}^{2+}$ fluorescent indicator gives an indication of the real $\mathrm{Ca}^{2+}$ sensed by the vescicles. This experimental method has been applied to the study of the large synapse of the auditory tract of the central nervous system, called calyx of Held. Moreover, it has been possible to build a minimal kinetic model for the process of the $\mathrm{Ca}^{2+}$ triggered vescicle release. Also, one can infer local $\mathrm{Ca}^{2+}$ signal waveform which is compatible with the experimental data on the time course and amplitude of release [SN00,SN05].

Most of the models treating the calcium triggered release issues present some methodological limitations. These models, and among them the calyx of Held model, use differential equations to describe the time course of $\left[\mathrm{Ca}^{2+}\right]$, the $\mathrm{Ca}^{2+}$ concentration $\left(\right.$ mole $\times$ liter $\left.^{-1}\right)$ interacting with the synaptic vescicles. This approach implies that $\left[\mathrm{Ca}^{2+}\right]$ is continuous, while it is clearly not [MBS]. For example, with a $\mathrm{Ca}^{2+}$ concentration of $10 \mu \mathrm{M}$ in a volume of $60 \mathrm{~nm}^{3}$ there is a single free ion. Another common assumption is that the binding of the $\mathrm{Ca}^{2+}$ to the release sensor of the vescicle does not affect the $\left[\mathrm{Ca}^{2+}\right]$ concentration [MBS]. Also this assumption is not properly adequate: considering that the dimensions 
of the vescicle diameters range in the interval 17-22 $\mathrm{nm}$, in a volume of $60 \mathrm{~nm}^{3}$ there could be few $\mathrm{Ca}^{2+}$ ions, and when some of them bind to the vescicle sensors, the number of calcium ions could change substantially. In these cases, the use of a stochastic approach appears to be much more appropriate.

The deterministic and the stochastic approach are the main formalisms to mathematically describe the time evolution of a chemical system which is spatially homogeneous. In the first approach, the time evolution of the average molecular populations of the chemical species present in a given volume is described by a system of coupled, first order differential equations, known as reaction rates equations. The second approach must be utilized when the fluctuation in the molecular population levels are important, for example when the numbers per unit volume of the molecular species involved are small. In the stochastic approach, the system is described by the so-called "master equation", which usually is intractable. A stochastic simulation algorithm has been proposed in [Gil77] to overcome these difficulties.

Recently, stochastic techniques have been also adopted in computer science to model quantitative aspects of interactive systems within concurrency theory. Concurrency theory aims to model the behaviour and the structure of systems composed of autonomous computational entities, which dynamically interact one with another, possibly reconfiguring the system itself. At the beginning, stochastic models have been used to study performance/time related properties, e.g. [Hil96]. The strong analogies between concurrent and living systems, "cells as computation" [RS02], has fostered the development of Systems Biology [Kit02, $\mathrm{CAD}^{+} 07$ ], a systemic approach to living system modeling. According to this metaphor, cells, molecules and biological "active" components, i.e. those capable of exhibiting a behaviour, are assimilated to computer processes, the computational units of a concurrent software system. Then, biological interaction corresponds to process communication. By communicating, processes may exchange information or synchronise themselves, i.e. they interact one with another. Finally, a biological experiment, or biological activity in general, has then a direct correspondence into computation. That is, biological processes can not only be simulated by in silico experiments, but also it is possible to formally reason about their computational models and infer properties of interest. Process calculi are a formalism to describe such models: systems are compositionally described in terms of suitable abstractions of their component behaviour. Several process calculi whose "operators" are oriented to describing different aspects of biological interaction have been proposed e.g. [NOMK99,PRSS04,RPS ${ }^{+}$04,Car04]. Some of these calculi have been equipped with stochastic semantics in order to study the quantitative evolutions of systems, e.g. [Pri04,PRSS04,LPQ ${ }^{+}$04,CGH06]. This approach benefits from conjugating the abstract and compositional algebraic models, the possibility of precisely describe their semantics and formally reasoning about them, and the quantitative analysis provided by stochastic semantics. Executable implementations of the calculi and analysis tools are provided. 
In this context, motivated by addressing some aspects of the functioning of neural synapses, we have developed a stochastic model of the calcium triggered release in the calyx of Held synapse.

Our work starts from a deterministic model presented in [SN00], from which we have derived a suitable stochastic model. This has subsequently been formalised in a variant of the Pi-calculus [Mil99], in which the behaviour of $\mathrm{Ca}^{2+}$ and vescicles has been described and composed to form the presynaptic terminal. Model development has benefited from the above mentioned features, like modular design, abstract representation of the component functioning and stochastic interpretation of the system dynamics. Then, in silico experiments have been carried out by means of the stochastic Pi-calculus simulator SPiM [PC04], which represents one of the most complete and expressive simulation environment for stochastic calculi currently available.

The developed model has been firstly tested against sensitivity and robustness, then tuned for our experiments of interest and, finally, used to investigate two aspects of the presynaptic plasticity mechanisms: paired pulse facilitation and short term depression.

Obtained results are coherent with those in literature and appears useful for better understanding and testing hypothesis on not fully understood parts of the two addressed issues.

Taking advantage of the compositionality of our approach, assemblying the more detailed neural model has been quite straightforward and, also, has suggested interesting directions for the enhancement of the expressive power of the representation language.

In the long term, we are interested further pursue the investigation along two complementary paths: from the biological viewpoint, we are interested to address models of synaptic plasticity, i.e. activity dependent change mechanisms, which are the bases of memory and learning processes, and to build more comprehensive stochastic models of synaptic functioning; form the computer science viewpoint, we aim at further developing the theory of concurrent biological interaction. In this sense, addressing a notion of (spatial) locality along the line suggested by the experiments would be a challenging task.

Synopsis The proposed model is described in Section 2, experiment results and a discussion on the model are presented in Section 3. Related work are discussed throughout the paper, while Section 4 contains concluding remarks. Preliminary results of our work have appeared in [BBCDar].

\section{A process calculi based stochastic model}

In this paper we have applied a stochastic model, based on the algorithm introduced in [Gil77], to describe the calcium triggered release mechanisms studied in the model system of the synapse calyx of Held. Our staring point was a phenomenological kinetic model, described in [SN00], in which five calcium binding steps and a cooperativity factor $b$ are needed for vescicle activation and release. 
The kinetic model parameters were computed by fitting experimental data, obtained by means of elevating the intracellular presynaptic $\left[\mathrm{Ca}^{2+}\right]$ in a controlled, homegeneous and step-like manner (calcium uncaging) [SN00]. We transformed the equations of the above cited model by utilising the relationship between the stochastic rate constants $(c)$ and deterministic rate constants $(k)$ [Kie02]. For reactions of the first order, $c=k$. For reactions of second order, the relationship becomes: $c=k /(N A \times V o l)$, where $N A$ represents the Avogadro's number and $V o l$ the volume of the reaction. Hence, in order to determine the values of the stochastic rate constants, we needed to estimate the value of $V o l$. Spatially, the calyx of Held is organized as a "parallel" arrangement of a large array of active zones, ranging from 300 to almost 700 [SF06]. Active zones, each containing up to 10 vescicles, are clustered in groups of about 10 of them, in a volume having a diameter of almost $1 \mu \mathrm{m}$. Each action potential activates all the active zones. Such particular morpho-functional organization of this synapse has allowed us to model a subunit of the presynaptic element, consisting of a cluster of 10 active zone, each containing 10 vescicles, in a volume of $0.510^{-15}$ liter. With this volume estimate, we have obtained the following values for the stochastic constants: $c_{\text {on }}=9 \times 10^{7} /\left(6.02 \times 10^{23} \times 0.5 \times 10^{-15}\right) s^{-1}=0.3 s^{-1}, c_{o f f}=9500 s^{-1}$, $\gamma=6000 s^{-1}$ and $b=0.25$, and the following numbers of $C a^{2+}$ ions: 300,3000 and 6000 , corresponding to molar concentrations $\left[\mathrm{Ca}^{2+}\right]$ of 1,10 and $20 \mu \mathrm{M}$. The equations of the stochastic model are:

$$
\begin{aligned}
& C a_{i}^{2+}+V \underset{c_{o f f} b^{0}}{\stackrel{5 c_{o n}}{\longrightarrow}} V_{C a_{i}^{2+}}+C a_{i}^{2+} \underset{2 c_{o f f} b^{1}}{\stackrel{4 c_{o n}}{\longrightarrow}} V_{2 C a_{i}^{2+}}+C a_{i}^{2+} \underset{3 c_{o f f} b^{2}}{\stackrel{3 c_{o n}}{\longrightarrow}} V_{3 C a_{i}^{2+}}+C a_{i}^{2+} \\
& V_{3 C a_{i}^{2+}}+C a_{i}^{2+} \underset{4 c_{o f f} b^{3}}{\stackrel{2 c_{o n}}{\longrightarrow}} V_{4 C a_{i}^{2+}}+C a_{i}^{2+} \underset{5 c_{o f f} b^{4}}{\stackrel{c_{o n}}{\longrightarrow}} V_{5 C a_{i}^{2+}} \stackrel{\gamma}{\longrightarrow} T
\end{aligned}
$$

where $\mathrm{Ca}_{i}^{2+}$ represents the number of intracellular calcium ions, $V$ the number of vescicles, $T$ the released vescicle.

Our simulations have confirmed the results obtained with the deterministic model: high sensitivity of vescicles to calcium concentrations [SN00]. Moreover, while several other synapses require a calcium concentration in the range of 100$300 \mu \mathrm{M}$ for triggering vescicle release [Sud04], it is known that the local calcium concentration can be much lower than $100 \mu \mathrm{M}$ in the calyx of Held [SN00]. Our results have also confirmed this result, by showing that concentrations as low as 1, 10 and $20 \mu \mathrm{M}$ are able to deplete the releasable pool in a few milliseconds. In the following, we will graphically report our results. Each figure consists of three pictures: on the left side the time course of $\mathrm{Ca}^{2+}$ is reported (together with the extrusion mechanism $(\mathrm{P})$ and its occupancy ( $\mathrm{CaP}$ ) when present, and the intermediate $\mathrm{Ca}^{2+}$ bindings); in the middle the same picture is reported in logarithmic scale so as to appreciate the intermediate states of calcium binding and vescicle activation (Vstar) and release (T); on the right side the focus is on activated vescicle (Vstar) and the total number of the transmitter released $(\mathrm{T})$. 
Figure 1 shows simulation results for the step-like calcium case with the following parameters: $V=100 ; C a^{2+}=6000 ; c_{o n}=0.3 ; c_{o f f}=9500 ; \gamma=6000$; $b=0.25$. It can be observed that the pool of vescicles is $80 \%$ depleted within 3 $m s$, coherently to the experimental findings [SN00].
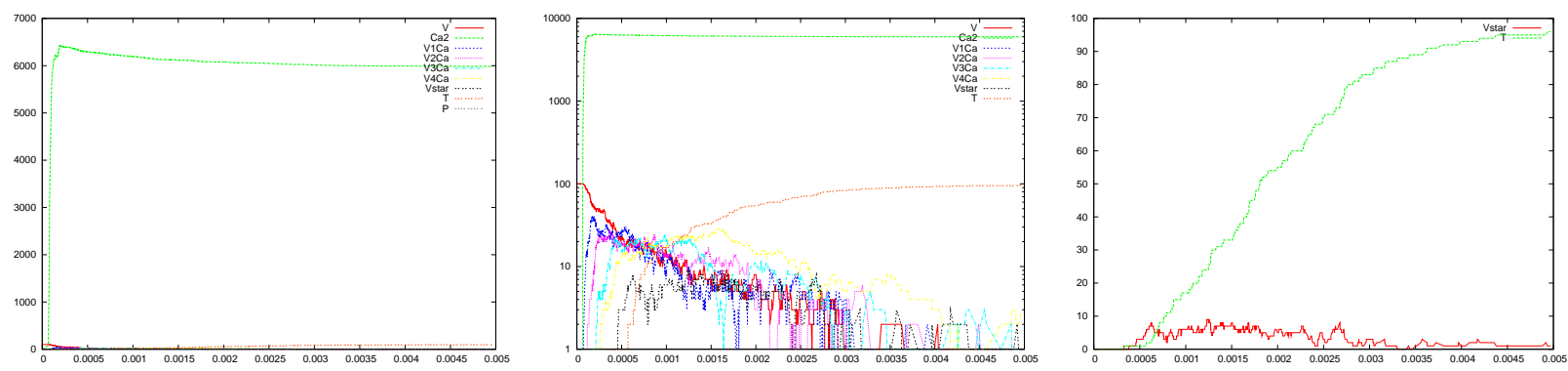

Fig. 1. Step-like calcium uncaging $\left(\mathrm{V}=100 ; \mathrm{Ca}=6000 ; \mathrm{C}_{\text {on }}=0.3 ; \mathrm{C}_{\text {off }}=9500 ; \gamma=6000 ; \mathrm{b}=0.25\right)$.
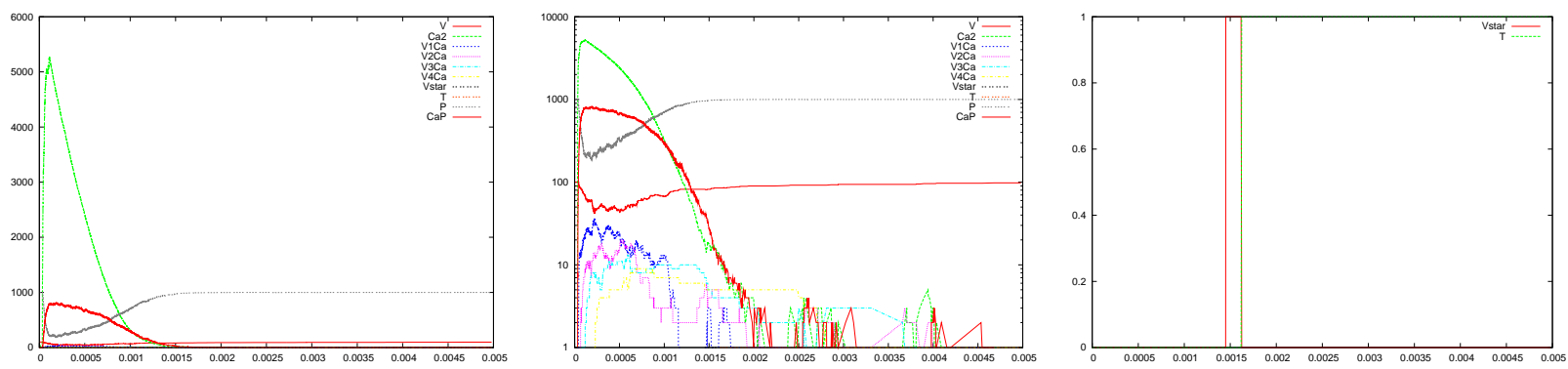

Fig. 2. Wave-like calcium uncaging ( $\left.\mathrm{V}=100 ; \mathrm{Ca}=6000 ; \mathrm{C}_{o n}=0.3 ; \mathrm{C}_{\text {off }}=9500 ; \gamma=6000 ; \mathrm{b}=0.25\right)$.

The experiments and models on $\mathrm{Ca}^{2+}$ uncaging [SN00] showed a high sensitivity of vescicle release in response to a uniform elevation of $\left[\mathrm{Ca}^{2+}\right]$ in the range $10 \mu \mathrm{M}$. It was not clear whether very short $\left[\mathrm{Ca}^{2+}\right]$ elevations are sufficient to induce a release similar to that induced during an action potential. A recent experimental work [BS05] has addressed this issue. A spatially uniform and very rapidly decaying $\left[\mathrm{Ca}^{2+}\right]$ transient, obtained by $\mathrm{Ca}^{2+}$ uncaging in the presence of added $\mathrm{Ca}^{2+}$ buffers, was induced in the presynaptic element of a calyx of Held synapse. This short-lived elevation (wave-like) of calcium concentration has been revealed to be able to trigger vescicle release. We have introduced in our model a simple mechanism of calcium extrusion utilized in a previously developed model [DMS94], adapting the rate constants to fulfill our needs:

$$
\mathrm{Ca}_{i}^{2+}+P \stackrel{c_{1}}{\longleftrightarrow} \mathrm{CaP} \stackrel{c_{3}}{\longrightarrow} \mathrm{Ca}_{o}^{2+}
$$

where $\mathrm{Ca}_{o}^{2+}$ is the extruded calcium, ${ }^{c_{2}} P$ is an abstraction of a pumping mechanism, $c_{1}=8 s^{-1}, c_{2}=25 s^{-1}$ and $c_{3}=10000 s^{-1}$. We have obtained a simulated calcium wave lasting about $1 \mathrm{~ms}$ and with a half width $0.5 \mathrm{~ms}$, conforming to the experimental requirements [BS05]. Such a kind of calcium wave with a peak 
value of about 6000 ions, corresponding to a peak calcium concentration of 20 $\mu \mathrm{M}$, can be seen on the left-side of Figure 2. In the right side of the same figure, the release of one vescicle can be observed. Considering that a whole presynaptic element can be made of about 70 of our simulated clusters, this implies that a single action potential, and accordingly a single calcium wave, is able to release a significant amount of vescicles. This is also along the line of the experimental findings [SN00,SN05,BS05].

For both models (step-like and wave-like calcium), we have performed a parameter variation study (sensitivity analysis). We have run simulations for different values of the number of vescicles (reference value 100, other values: 10, 50, 200 and 500), the number of calcium ions $\mathrm{Ca}^{2+}$ (reference values 300, 3000 and 6000 , other values: $12000,18000,24000$ ), the stochastic coefficients $c_{\text {on }}$ (reference value 0.3 , other value: $0.1,0.2,0.4$ and 0.5 ), $c_{o f f}$ (reference value 9500 , other values: $5500,7500,11500$ and 13500 ), $b$ (reference value 0.25 , other values: $0.1,0.2,0.3$ and 0.4 ) and $\gamma$ (reference value 6000 , other values: $2000,4000,8000$ and 10000).

One of the results of this analysis is that the forward coefficient $c_{o n}$ seems to have a critical role: higher values of this coefficient correspond to a faster release, in the step-like case, and to a switch from no-release to a consistent release, in the wave-like case. For the step-like case, we showed that a simple variation of the coefficients $c_{o n}$ and $b$ changes the dynamics of the release processes in an unpredictable manner.

This kind of experiments are of interest when addressing the problem of the variations in the release rate, one of the still obscure phenomena which have been observed about vescicle release. These variations have been explained by the recruitment of new vescicles within the same active zone or by a different sensitivity to calcium ions of the vescicle belonging to same cluster. Our kind of analysis might give some contributions to the debate on the interpretation of these controversial experimental data [SN00,SN05,BS05].

\subsection{Neuro-processes}

In order to illustrate the main features of the formal model used to stochastically represent the behaviour of the calyx of Held synapse, we briefly sketch some of its parts. Excerpts from the model, viz. its implementation for the SPiM stochastic interpreter [PC04], are in Figure 3. The representation language models interaction as pairs of input/output actions over the same communication channel $(? c / ! c)$. These atomic actions can be composed in a sequence (;) or in alternative choices $($ ?c or ?d) so as to form a process $(\mathrm{p}()=\ldots)$. Processes can run in parallel $(\mathrm{p}() \mid \mathrm{q}())$.

Initially, the length of the simulation is set (here $0.005 s$ ), then some stochastic parameters are defined. Communication channels can be (dynamically) created by means of the new command and have associated a stochastic rate (this and the current quantities of reactants determine the probability of a reaction "happening" through the channel). A calcium ion $(\mathrm{ca}())$ can interact with a vescicle $(\mathrm{v}())$ over channel vca with rate con $5=1.5$ (beyond being able to do other 
things). After this communication, ca() disappears and $\mathrm{v}()$ becomes v_ca(), representing the binding of the two. This realises a second order reaction. First order reactions are modeled as interactions with a single dummy molecule (so as not to alter stochastic dynamics). For instance, v_ca() can then either accept other calcium bindings or degradate back to an unbound vescicle by communicating through bvca with Dv_ca(), which restores ca() and itself. So far, the system has been described by specifying simple atomic behaviour, basically corresponding to chemical reactions, and then composing them together.

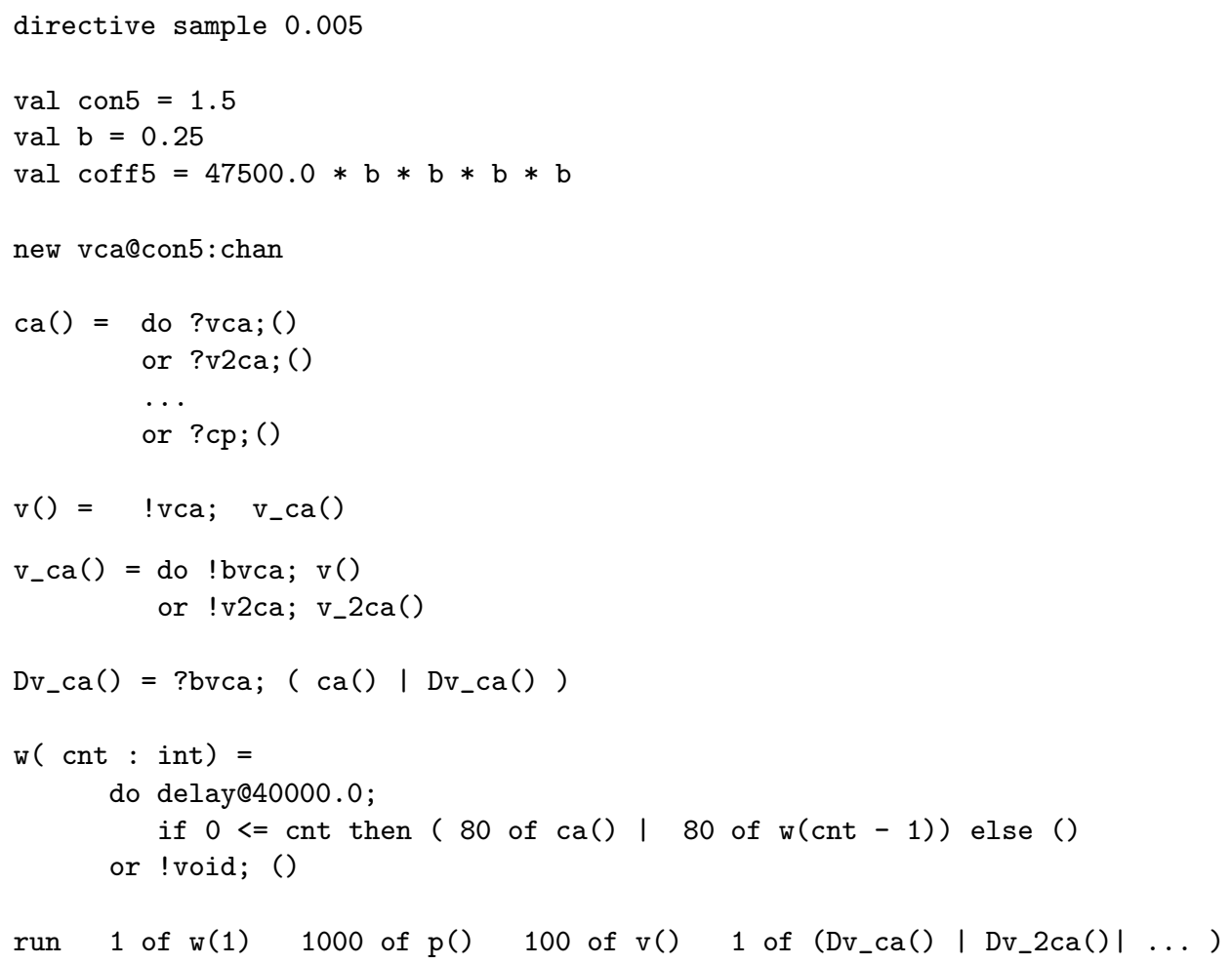

Fig. 3. The calyx of Held SPiM code.

The parametric process $\mathrm{w}$ (cnt:int) allows us to suitably modulate the calcium wave. After a stochastic delay, it replicates 80 copies of itself in parallel with $80 \mathrm{ca}()$ if its integer parameter cnt is positive, otherwise it dies. This realises an exponential growth, which can be controlled by the delay rate and the parameter in its rapidity and quantity. Finally, the initial state can be populated specifying how many molecules of each specie are present (1 wave, 1000 pumping molecules, 100 vescicles and 1 copy of the needed dummy molecules).

\section{Results and model evaluation}

Exploiting the developed model, we have addressed some open issues regarding the presynaptic mechanisms for $\mathrm{Ca}^{2+}$ triggered vescicle release. More specifically, 
we have considered temporal and spatial aspects of the release which appear intertwined and relevant for synaptic plasticity. On the one hand, we have studied the behaviour of the synaptic terminal in the presence of a train of action potentials occurring within short temporal intervals. We report on an analysis of two $\mathrm{Ca}^{2+}$ waves and discuss how this is related to facilitation, i.e. a form of activity dependent enhancement of the synaptic strength. On the other hand, we have tried to find a suitable model of the spatial (and functional) distribution of vescicles within the presynaptic terminal. This has been done in order to support the verification of competing hypotheses on the mechanisms ruling short-term synaptic depression. With this aim we have analysed the relationship between the measured pattern of the time course of release and the possible existence of two pools of different vescicles in the active zone [FNS03,SSJN05,WSN99,SSN02a].

In order to tune the model for these two experiments, we have beforehand run a series of simulations (not reported here) with the aim of both evaluating the variance of the results of different runs, and of tuning the model to the hypotheses of the experiments. In particular, the hypotheses about vescicle distribution has required us to revise the assumption on the number, and also the behaviour, of vescicles. This is needed to obtain average release values during a single action potential that are coherent with the experimental findings in the scenaria of interest. In particular, setting the number of the readily releasable vescicles $(V)$ to 50 and increasing their propensity to calcium bounding $\left(c_{o n}\right)$ to $0.4 s^{-1}$ has resulted in an average number of released vescicles of about 2 , which is about $4 \%$ of the (readily releasable) vescicle pool, adherent to the experimental findings [FNS03].

The compositionality of the chosen representation language has permitted us to easily embed the new features in the model by means of modular changes. Moreover, the challenge of dealing with the illustrated spatial and temporal aspects seems to suggest fruitfully directions for extending the expressiveness of the representation language. Both these aspects are discussed in Section 3.3.

With this more detailed model, we have hence studied the phenomenon of synaptic transmission called paired pulse facilitation (Section 3.1) and synaptic depression during sustained depolarization of the synapse (Section 3.2), which are examples of short-term synaptic plasticity still puzzling neuroscientists.

\subsection{Paired pulse facilitation}

The term synaptic facilitation indicates a form of activity-dependent synaptic enhancement observed in several synapses, in which the synaptic strength increase during a train of action potentials [FNS03,ZKS04]. In general, facilitation depends by pre and post -sinaptic mechanisms. In this paper, we focus on the presynaptic side of facilitation. It is generally accepted that the intracellular $\mathrm{Ca}^{2+}$ remaining from previous activity (the so-called residual $\mathrm{Ca}^{2+}$ ) causes facilitation, but the mechanisms that make this happens are still not clearly understood [Sud04]. In general, the residual $\mathrm{Ca}^{2+}$ is less than $1 \mu \mathrm{M}$ (in our model this correspond to less than $300 \mathrm{Ca}^{2+}$ ions) and for most synapses the 
local $\left[\mathrm{Ca}^{2+}\right]$ increase needed for release is between 100 and $300 \mu \mathrm{M}$. This implies that, typically, the residual $\mathrm{Ca}^{2+}$ is not sufficient to induce facilitation.

Some other underlying mechanisms for facilitation have been proposed, such as, just to cite some, the permanence of $\mathrm{Ca}^{2+}$ bound to the high affinity binding sites of the secretory machinery, $\mathrm{Ca}^{2+}$ buffer saturation or an increased size of the readily releasable (see Section 3.2) pool [FNS03].
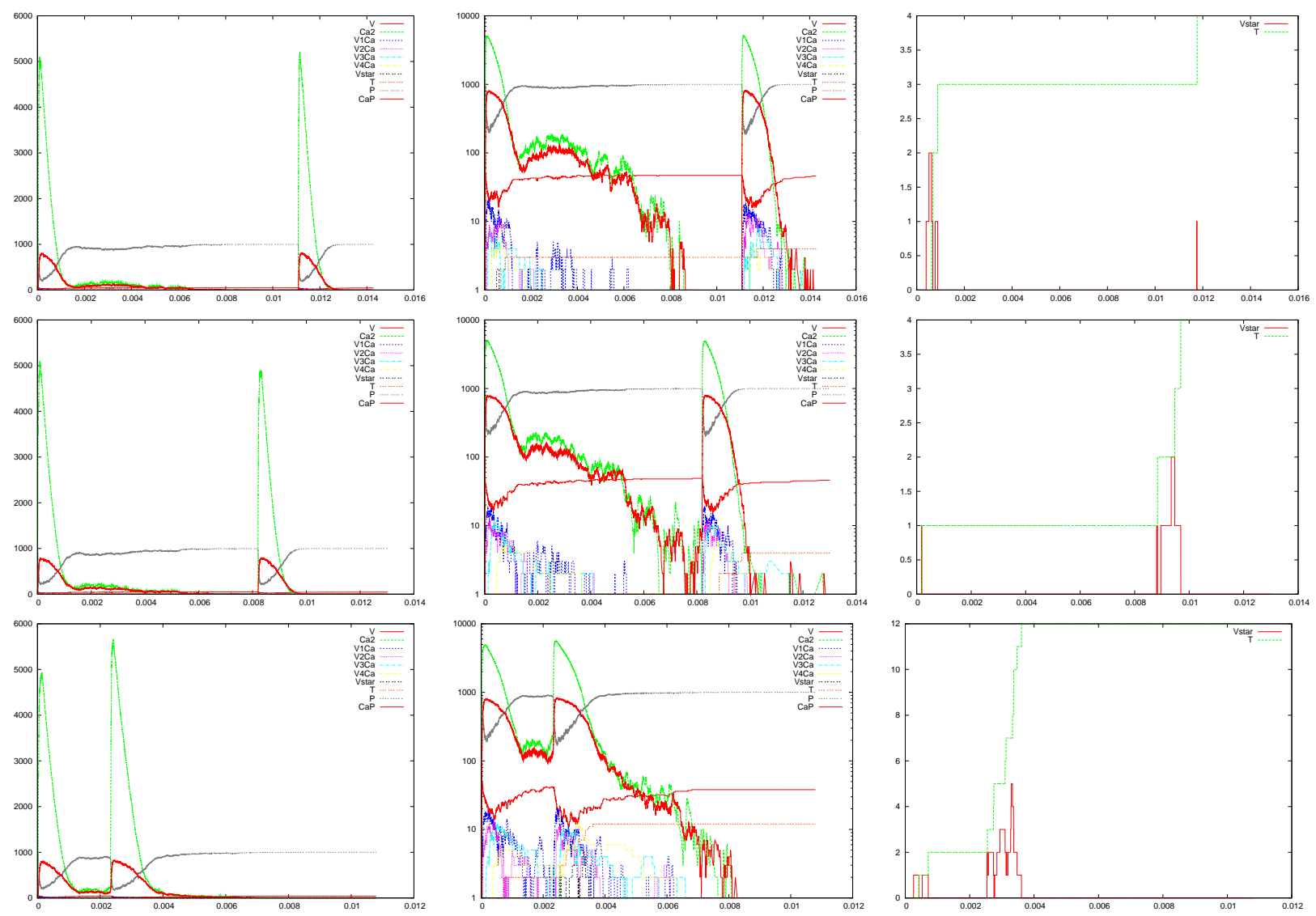

Fig. 4. Two wave-like calcium pulses at different intervals $\left(\mathrm{V}=50 ; \mathrm{C}_{\text {on }}=0.4\right)$.

We have run a series of simulations in which we have used a double $\mathrm{Ca}^{2+}$ wave in order to study facilitation. We report in Figure 4 a small sample selection of our simulations with varying time delays between waves, each row represents a simulation. In the left pictures, and correspondingly in the others, it is possible to appreciate the occurrence of the two waves with varying delays. From upper to lower row, the time interval betwee $\mathrm{Ca}^{2+}$ wave decreases (12, 8 and $2 \mathrm{~ms}$ ) and it can be observed that for smaller delay the amount of release due to the second wave increases notably. 
The possible underlying mechanisms could be suggested by observing the central column of Figure 4. Just before the second calcium wave develops, the amount of residual $\mathrm{Ca}^{2+}$ is bigger when the delay decreases. This causes a concomitant increase of the release.

At the same time, it seems that other parts of the release machinery could be involved in facilitation, such as the occupancy of $\mathrm{P}$ or the intermediate steps of vescicle binding. In our simulations, also these parts seem to be influenced by residual $\mathrm{Ca}^{2+}$, as can be seen, for instance, by looking at the level of occupancy of $\mathrm{P}$. Hence, the residual $\mathrm{Ca}^{2+}$ seems to have a central role in the facilitation process, even at very low concentrations.

Summing up, our simulations support the hypothesis that in calyx of Held, facilitation is likely due to residual $\mathrm{Ca}^{2+}$ and to occupancy of $\mathrm{Ca}^{2+}$ buffers, which are cellular elements which control, by reducing it, the $\mathrm{Ca}^{2+}$ concentration. Our simulations show that our model is consistent with the idea that a very low level of residual $\mathrm{Ca}^{2+}$ might account for the particular form of short-term synaptic plasticity named paired pulse facilitation [FNS03,ZKS04].

\subsection{Short-term synaptic depression}

It is known by experimental data that the vescicles of the active zone can be divided in two virtually separated and equally populated pools: one consisting of so-called readily releasable vescicles and the other one of so-called reluctantly releasable vescicles [Neh06,SSJN05,WSN99].

During a single action potential discharge, which in our model is mimicked by a single calcium wave, the vescicles released belong to the readily releasable pool. The amount of release corresponds to about $4 \%$ of the readily releasable pool, hence in our model to 2-3 vescicles [FNS03]. Whereas, when the synapse is depolarized to elicit maximal $\mathrm{Ca}^{2+}$ influx, all the vescicles of the active zone are released (readly and reluctantly releasable) and the synapse active zone is completely depleted. In this case the time course of the release process is charachterized by two time constants, which are about 3 and $30 \mathrm{~ms}$, during which the readily and reluctant vescicle are released, respectively [Neh06,SSN02b]. It is also known that the reluctant vescicles are replaced much more rapidily than the readily releasable ones.

The precise mechanisms through which this form of release happens are still debated. For some cells, such as the chromaffin cells, it is likely that the reluctant vescicles differ, in their intrinsic kinetics, from the readily releasable ones [Sor04].

For the calyx of Held synapse, it has been suggested, by means of a qualitative model, that the reluctant vescicles are precursors of the rapidly releasing ones and that they become readily releasable by laterally moving toward the readily releasable pool [Neh06]. Based on these suggestions, we have built a stochastic multi-pool model of the possibile mechanisms underlying short-term synaptic depression. The coefficients have been in part obtained from the literature and in part obtained by trails and errors, because generally unknown. 
In this stochastic model, one pool represents the readily releasable vescicles (docked to the active zone), which are released according to the model previously developed and whose starting number has been set to 50 .

The reluctant vescicles (whose starting number is 50) go back and forth between two other pools, in which they are undocked (Rct_V), i.e. not releasable, and docked $\left(R_{-} V\right)$, i.e. releasable, respectively. The undocked reluctant are rapidily replenished from a forth pool (Inf_V) of vescicles, which represents the reservoir of the reluctant vescicles:

$$
\text { Inf_V } \underset{1 s^{-1}}{\stackrel{10 s^{-1}}{\longleftrightarrow}} R c t_{-} V \underset{0.1 s^{-1}}{\stackrel{2.5 s^{-1}}{\longleftrightarrow}} R-V+C a_{i}^{2+} \underset{c_{o f f} b^{0}}{\stackrel{5 c_{o n}}{\longrightarrow}} \cdots \underset{c_{o f f} b^{4}}{\stackrel{c_{o n}}{\longrightarrow}} R \_V_{5 C a_{i}^{2+}} \stackrel{\gamma}{\longrightarrow} T
$$

The results of the simulations are reported in Figure 5 for the case of 300 and 1000 In $f_{-} V$ vescicles, respectively. Here, the left picture shows calcium dynamics as usual, the center one reports calcium again and the four kind of vescicles and their activation and release in logarithmic scale, while the rightmost picture reports again activated vescicles and the corresponding cumulative release. In both simulations, the continuous depolarization was mimicked by a step like calcium wave. In the central column it can be seen the synchronous release (darker, within the first $3 \mathrm{~ms}$ ) and the asynchronous one (grey in the picture). In the right pictures, it is very clearly visible the double slope of the cumulative release curves.

The two right pictures are qualitatively similar: it can be observed a fast release (during about $3 \mathrm{~ms}$ ) of about 50 vescicles, followed by a slower release.
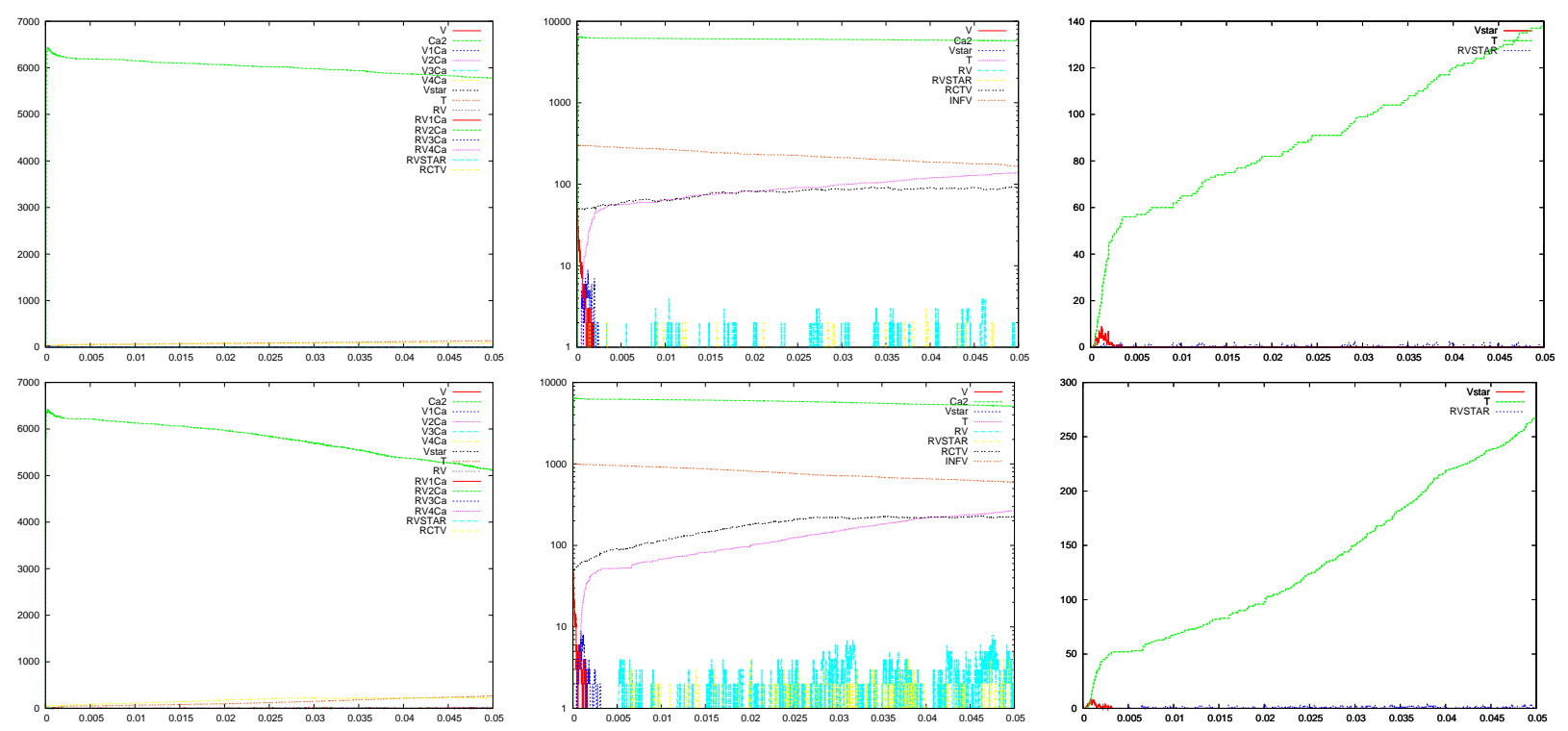

Fig. 5. Multipools vescicle activation $\left(\mathrm{C}_{\text {on }}=0.4 ; \mathrm{V}=50 ; \mathrm{R}_{-} \mathrm{V}=0 ; \mathrm{Rct}_{-} \mathrm{V}=50 ;\right.$ Inf_V=300(top); Inf_V=1000(bottom)). 
In the first row of Figure 5, during the slower release, 50 vescicles (rather than 150 of the second row) are released in about $30 \mathrm{~ms}$, which is most adherent to the experimental findings.

This model helps to clarify the mechanisms of delocalization of vescicles in the active zone, helps to determine some unknown parameters (stochastic coefficients, number of vescicles) and reproduces some experimental observations. It must be noted that it is a partial description of the complex and still poorly understood short-term synaptic depression process [Sud04]. Nevertheless, it suggests that the stochastic constants cannot be effectively constant: they would need to be modulated during the ongoing process of release, otherwise the movements of the vescicle would be out of equilibrium condition during the rest state of the synapse. This last condition could explain the discrepancy between the values of some coefficients utilized in the model and the corresponding values found in the literature, for example the value 2.5 for the coefficient was indicated to be 0.25 .

\subsection{Extending the stochastic model and further considerations}

The core Calyx of Held stochastic model, i.e. the one featuring step-like or (one) wave-like $\mathrm{Ca}^{2+}$ uncaging, has been easily extended in order to support the presented experiments.

The generation of a second wave trivially consists of running a second identical wave-generator process (see Figure 3). Calling the second generator with a different name (w2(1)) allows us to more easily distinguish the two pulses, when needed. Moreover, in order to control the delay of the second wave, a process that activates the generator after a parametric nondeterministic delay has been introduced: snd_w ()$=$ delay@125.0; w2(1). Although varying the delay parameter has allowed us to simulate a wide range of delays (some of which reported in Figure 4), this experiment has suggested the utility of a more precise time control over processes. In general, there might be several cases in which one would like to be able to express something like

$$
\text { run } 1 \text { of } \mathrm{w}(1) \text { at } 0.002
$$

meaning that the stochastic process $\mathrm{w}_{(1)}$ is supposed to start at about (in a sense to be specified according to the stochastic nature of the approach) time 0.002 of the simulation. This issue is under study.

The presence of residual calcium has been analogously modeled by modularly adding a generative process. Tuning the recursive branching factor and the delay parameter (e.g. 80 and 4000 for the case of w (cnt) in Figure 4) has allowed us to control the amount of $\mathrm{Ca}^{2+}$ and the time interval needed by the extrusion mechanism to clear it.

Besides the temporal aspects, also the spatial ones have needed to be engineered so that the desired behaviour is suitably modeled. According to the multi-pool hypothesis we have distinguished the reluctant vescicles of replenishing pools (inf_v( )) from those undocked (rct_v()) and those docked $(\operatorname{rv}())$ : 


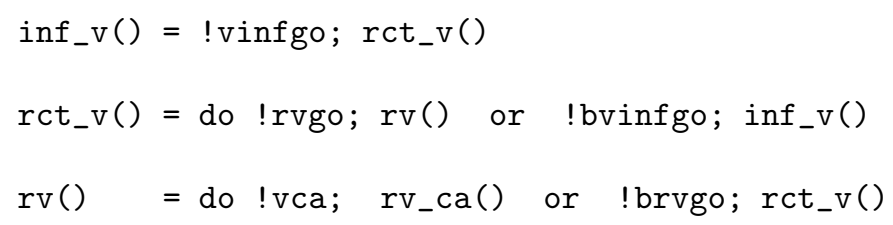

Accordingly to the presented model, the behaviour of $\mathrm{rv}()$ is undistinguishable from that of the readily releasable $\mathrm{v}()$ with respect to calcium binding. Indeed, note that once docked, rv() interacts with ca() through the same channel vca used by $\mathrm{v}()$. This preserves correctness of the model since, coherently with the interpretation of docked $\mathrm{rv}()$, both kind of vescicles participate to calcium dynamics (and both concur, insisting on the same communication channels, to the Gillespie-based stochastic dynamics [PC04]). Such name distinction allows us to clearly mark fast and slow vescicles in Figure 5.

Although this reading of a "spatially" distributed (over different pools or classes of processes) behaviour has allowed us to perform coherent simulations, it seems worth investigating more expressive spatial primitives. A starting point is naturally represented by location-aware calculi a là Ambient calculus, like the Brane Calculi [Car04]. However, the problem of understanding the relation between stochastic dynamics and spatial information, e.g. how the traversal of an axon modifies signal strength, and hence its "interaction capability", is still open. Along this line goes the possibility of dynamically modifying the stochastic rates during a stochastic run, as advocated in Section 3.2.

\section{Conclusion}

The presented results are encouraging about the validity of the stochastic approach in studying the synaptic processes, which consist of many discrete-like events and involve arrays of vescicles and hundreds of different molecules. Many of these molecules have roles in the process of synaptic transmission which still are not fully understood [Sud04]. We started by studying the release process by using data of a simplified experimental model, in which the concentration of $\mathrm{Ca}^{2+}$ was controlled and homogeneous. Under these hypotheses, the issue of spatial locality could not have been considered. We have extended the model in order to study events taking place during prolonged neural activity. To this aim, we added more details on vescicle trafficking and cluster compartimentalisation [Neh06], and we studied short-term synaptic plasticity, facilitation and synaptic depression. Embedding these processes in the model might shed some light on the ways the nervous system processes and stores information. In order to support these, and others, developments the underlying process calculus might be extended, too. Surely, the problem of expressing locality, already addressed by several calculi, could be valuably addressed within a stochastic viewpoint. 


\section{References}

[BBCDar] A Bracciali, M Brunelli, E Cataldo, and P Degano. In silico stochastic simulation of $\mathrm{Ca}^{2+}$ triggered synaptic release. In Network Tools and $\mathrm{Ap}$ plication in Biology (NETTAB07), 2007 (To appear).

[BS05] J H Bollmann and B Sakmann. Control of synaptic strength and timing by the release-site $\mathrm{ca}^{2+}$ signal. Nature Neuroscience, 8:426-434, 2005.

$\left[\mathrm{CAD}^{+}\right.$07] M Cassman, A Arkin, F Doyle, F Katagiri, D Lauffenburger, and C Stokes. System Biology - International Research and Development. Springer, The Netherlands, 2007.

[Car04] L Cardelli. Brane calculi-interactions of biological membranes. In V Vincent and V Schachter, editors, Proceedings of Computational Methods in Systems Biology, pages 257-280. Springer, 2004.

[CGH06] M Calder, S Gilmore, and J Hillston. Modelling the influence of rkip on the erk signalling pathway using the stochastic process algebra pepa. Transactions on Computational System Biology VII, LNBI 4230:1-23, 2006.

[DMS94] A Destexhe, Z F Mainen, and T J Sejnowski. Synthesis of models for excitable membrane, synaptic transmission and neuromodulation using a common kinetic formulation. The Journal of Computational Neuroscience, 1:195-231, 1994.

$\left[\mathrm{FGJ}^{+}\right.$06] M Frotscher, E Gundelfinger, P Jonas, E Neher, and P Seeburg. The most important recent advances in synsapse research from my point of view and what remains to be done. Cell Tissue Res, 326:203-204, 2006.

[FMWT02] C P Fall, E S Marland, J M Wagner, and J J Tyson, editors. Computational Cell Biology, New York, 2002. Springer.

[FNS03] F Felmy, E Neher, and R Schneggenburger. Probing the intracellular calcium sensitivity of transmitter release during synaptic facilitation. Neuron, 37:801-811, 2003.

[Gil77] D T Gillespie. Exact stochastic simulation of coupled chemical reactions. The Journal of Physical Chemistry, 81:2340-2361, 1977.

[Hil96] J Hillston. A Compositional Approach to Performance Modelling. Cambridge University Press, Cambridge, 1996.

[Kie02] A M Kierzek. Stocks: Stochastic kinetic simulations of biochemical system with gillespie algorithm. Bioinformatics, 18:470-481, 2002.

[Kit02] H Kitano. Systems biology: a brief overview. Science, 295(5560):1662-1664, 2002.

[KS98] C Koch and I Segev, editors. Methods in Neuronal Modeling, Cambridge, 1998. The MIT Press.

[LPQ ${ }^{+}$04] P Lecca, C Priami, P Quaglia, B Rossi, C Laudanna, and G Costantin. A stochastic process algebra approach to simulation of autoreactive lymphocyte recruitment. SIMULATION: Trans. of the society for modelling and simulation international, 80(4):273-288, 2004.

[MBS] C J Meinrenken, J G G Borst, and B Sakmann. The hodgkin-huxley-katz prize lecture. local routes revisited: the space and time dependence of the ca signal for phasic transmitter release at the rat calyx of held. in press.

[Mil99] R Milner. Communicating and Mobile Systems: The $\pi$-Calculus. Cambridge University Press, 1999.

[Neh06] E Neher. A comparison between exocytic control mechanisms in adrenal chromaffin cells and a glutamatergic synapse. Pflugers Arch - Eur J Physiol, 453:261-268, 2006. 
[NOMK99] M Nagasaki, S Onami, S Miyano, and H Kitano. Bio-calculus: its concept and molecular interaction. Genome Informatics, 10:133-143, 1999.

[PC04] A Phillips and L Cardelli. A correct abstract machine for the stochastic pi-calculus. In Proceedings of Bioconcur'04. ENTCS, Elsevier, 2004.

[Pri04] C Priami. Stochastic $\pi$-calculus. The Computer Journal, 36(6):578-589, 2004.

[PRSS04] C Priami, A Regev, E Shapiro, and W Silvermann. Application of a stochastic name-passing calculus to representation and simulation of molecular processes. Theoretical Computer Science, 325(1):141-167, 2004.

[RBM] Rediscovering Biology - Molecular to Global Perspectives - Neurobiology. http://www.learner.org/channel/courses/biology/support/10_neuro.pdf.

$\left[\mathrm{RPS}^{+}\right.$04] A Regev, E Panina, W Silverman, L Cardelli, and E Shapiro. Bioambients: An abstraction for biological compartements. Theoretical Computer Science, 325(1):141-167, 2004.

[RS02] A Regev and E Shapiro. Cellular abstractions: Cells as computation. $\mathrm{Na}$ ture, 419:343, 2002.

[SF06] R Schneggenburger and I D Forsythe. The calxy of held. Cell Tissue Res, 326:311-337, 2006.

[SN00] R Schneggenburger and E Neher. Intracellular calcium dependence of transmitter release rates at a fast central syanpse. Nature, 46:889-893, 2000.

[SN05] R Schneggenburger and E Neher. Presynaptic calcium and control of vescicle fusion. Current Opinion in Neurobiology, 15:266-274, 2005.

[Sor04] JB Sorensen. Formation, stabilization and fusion of the readily releasable pool od secretory vescicles. Pflugers Arc - European Journal of Neuroscience, 448:347-362, 2004.

[SSJN05] t Sakaba, A Stein, R Jahn, and E Neher. Distinct kinetic changes in neurotransmitter release after snare protein cleavage. Science, 309:491-494, 2005.

[SSN02a] T Sakaba, R Schneggenburger, and E Neher. Estimation of quantal parameters at the calyx of held synapse. Neuroscience Research, 44:343-356, 2002.

[SSN02b] R Schneggenburger, T Sakaba, and E Neher. Vescicle pools and short-term synaptic depression: lessons from a large synapse. TINS, 25:206-212, 2002.

[Sud04] T C Sudhof. The synaptic vescicle cycle. Annual Review of Neuroscience, 27:509-547, 2004.

[WSN99] S Weis, R Schneggenburger, and E Neher. Properties of a model of $\mathrm{Ca}^{2+}$ -dependent vescicle pool dynamics and short term synaptic depression. Biophysical Journal, 77:2418-2429, 1999.

[ZKS04] R S Zucker, D M Kullmann, and T L Schwartz. Release of neurotransmitters. In J H Byrne and J L Roberts, editors, From Molecules to Networks - An Introduction to Cellular and Molecular Neuroscience, pages 197-244. Elsevier - Academic Press, 2004. 